\section{Clinical response of clozapine as a treatment for delirious mania}

\author{
Nabeel Wahid, ${ }^{1}$ Garwin Chin, 1 \\ Andia H. Turner, ${ }^{2}$ Alexis Seegan ${ }^{2}$ \\ 1School of Medicine, and 2Department \\ of Psychiatry, University of California, \\ Irvine, Orange, CA, USA
}

\begin{abstract}
Delirious mania is an understudied psychiatric disorder with a mortality rate as high as $75 \%$. Previous case studies suggest that electroconvulsive therapy (ECT) may be an effective treatment for delirious mania, though this procedure may not always be a viable option. We describe the case of a 20-year old patient, with no previous psychiatric history, who developed delirious mania over the course of four months. ECT was not a viable option for this patient due to his religious beliefs, so alternative treatment modalities were explored. After failing trials of risperidone and olanzapine, significant improvements in symptoms were exhibited with a trial of clozapine. We propose that clozapine may be an effective option in cases of delirious mania, when ECT is not a viable option. Additional research is still necessary to understand the pathology of this condition and potential treatment modalities.
\end{abstract}

\section{Introduction}

Delirious mania is a potentially fatal yet understudied condition with no clear consensus of clinical characteristics and symptoms. ${ }^{1,2}$ It is not described in the most recent edition of the Diagnostic and Statistical Manual of Mental Disorders (DSM V) and criteria used for diagnosis is mostly based on a series of case reports. Symptoms include emotional liability, delusions, insomnia, disorientation, acute onset of grandiosity, altered consciousness, incontinence/inappropriate toileting, and hyper sexuality.2-4 Given its multi-faceted symptomology, this disease may be confused with other psychiatric conditions such as bipolar disorder, psychosis, malignant catatonia, or delirium. 5 Reports have demonstrated that trials of antipsychotics have minimal symptomatic improvement and can lead to exacerbation of the catatonia. ${ }^{6}$ Without prompt recognition and treat- ment, delirious mania can progress and potentially become life-threatening, ${ }^{1}$ with mortality rates as high as $75 \%{ }^{4}$ By far, the most effective described treatment for delirious mania has been electroconvulsive therapy (ECT), ,3-5-8 however, this case report describes a potential pharmaceutical alternative in cases when ECT is not a feasible option.

\section{Case Report}

A 20-year-old male, with no previous psychiatric history, was admitted for severe catatonia, mutism, and insomnia. His symptoms started four months prior with increased anxiety and depression. Although some improvement of catatonic features were seen with high doses of lorazepam, the patient appeared delirious with waxing and waning episodes of disorientation, disorganized speech and behavior, hyper-religiosity, hypersexuality, and paranoid thoughts. He had multiple episodes of encopresis and enuresis, which together with his other described symptoms appeared consistent with a diagnosis of delirious mania. Neuroleptic malignant syndrome was excluded due to lack of hyperthermia, autonomic instability and onset of symptoms prior to exposure to neuroleptic drug. Other considerations included bipolar disorder because the patient demonstrated poor sleep, distractibility, and erratic behavior. However, his presentation included multiple other symptoms and did not remain consistent enough to be attributed to bipolar disorder. Psychosis was considered, and was likely a component of what the patient was experiencing, however he had an unusual presentation that included additional symptoms unexplained by pure psychosis.

Trials of risperidone and olanzapine did not improve his symptoms. ECT was strongly recommended but was ultimately avoided due to the religious beliefs of patient's family and prolonged legal process for obtaining a court order. As such, clozapine was added to his regimen of lorazepam and valproate, and was titrated up to $450 \mathrm{mg}$ per day over the course of 21 days. The daily dosing regimen for clozapine and lorazepam are included in Figure 1 (A and B respectively). The patient showed significant improvement in his orientation and thought process by day 27 of clozapine treatment (day of hospitalization 41). He was deemed safe for discharge 45 days after admission. Although he continued to show some emotional labiality, the combination
Correspondence: Nabeel Wahid, 1001 Health Sciences Rd, Irvine, CA 92617, USA.

E-mail: n.wahid@uci.edu

Key words: clozapine, delirious mania, electroconvulsive therapy.

Contributions: the authors contributed equally.

Conflicts of interest: the authors declare no potential conflict of interest.

Received for publication: 12 April 2017.

Revision received: 1 June 2017.

Accepted for publication: 2 June 2017.

This work is licensed under a Creative Commons Attribution-NonCommercial 4.0 International License (CC BY-NC 4.0).

CC Copyright N.Wahid et al., 2017

Licensee PAGEPress, Italy

Mental Illness 2017; 9:7182

doi:10.4081/mi.2017.7182

of clozapine, valproate, and lorazepam significantly improved his symptoms of delirium, mania, and catatonia.

\section{Discussion}

A recent systematic review of ECT found it to be an effective treatment option for all forms of catatonia, including malignant catatonia and delirious mania, even in cases that are refractory to benzodiazepines. ${ }^{7}$ Responsiveness to ECT in cases of delirious mania have been shown in as few as three ECT sessions. ${ }^{3}$ Additionally, given that delirious mania is difficult to diagnose and has been found in many patients with a history of bipolar disorder, ${ }^{2}$ ECT could be used to treat multiple pathologies if the presentation is unclear. ${ }^{7}$

Nonetheless, ECT is not always accepted by patients or available by practitioners. This case demonstrates an alternative medication regimen that led to considerable improvement in symptoms. Although it could be argued that the patient may not have capacity if they are experiencing delirious mania (and an incapacitated courtordered ECT could be pursued), it may not be in the best interest of the patient if his or her wishes are known prior to their illness.

As far as alternative treatment options for delirious mania, the literature is limited mostly to case reports. One case of delirious mania exhibited considerable improvement in symptoms with quetiapine, with the patient discharged after 29 days. ${ }^{4}$ Another 
case described improvements with olanzapine over the course of 2 months after ECT was suspended due to technical issues. ${ }^{9}$ In our patient with delirious mania, olanzapine was ineffective and quetiapine was not trialed because the patient had failed two trials of other atypical antipsychotics. With limited additional treatment options shown in the literature, a trial of clozapine was started. Although a patient with delirious catatonic mania in a previous case report continued to have deterioration in mental status with clozapine treatment, ${ }^{8}$ our patient had considerable improvement after 27 days of treatment with clozapine. The slow titration was secondary to the patient experiencing significant side effects, and thus other patients may be able to reach a therapeutic dose in a shorter time period.

Recent studies have suggested that in patients with treatment resistant schizophrenia, clozapine has greater effectiveness than standard antipsychotics. ${ }^{10}$ Mechanistically, it is likely that the therapeutic profile of clozapine is mostly due to its antagonism to the 5-HT2A and D4 receptors along with weak D2 antagonism. ${ }^{11}$
As such, further research may consider targeting these receptors in developing new pharmaceutical treatment modalities for delirious mania.

\section{Conclusions}

Additional research is necessary to understand why clozapine is effective in some cases of delirious mania but not others. Given the nature and rarity of this disease, there are practical and ethical challenges to conducting randomized clinical trials that compare different treatment modalities. Nonetheless, clinical practice would benefit from additional literature to better understand the course of delirious mania and the optimal treatment modalities for individual patients. As case reports are the primary source for information about this illness, increasing available data is the next step in understanding the pathology underlying delirious mania and creating future treatment guidelines.
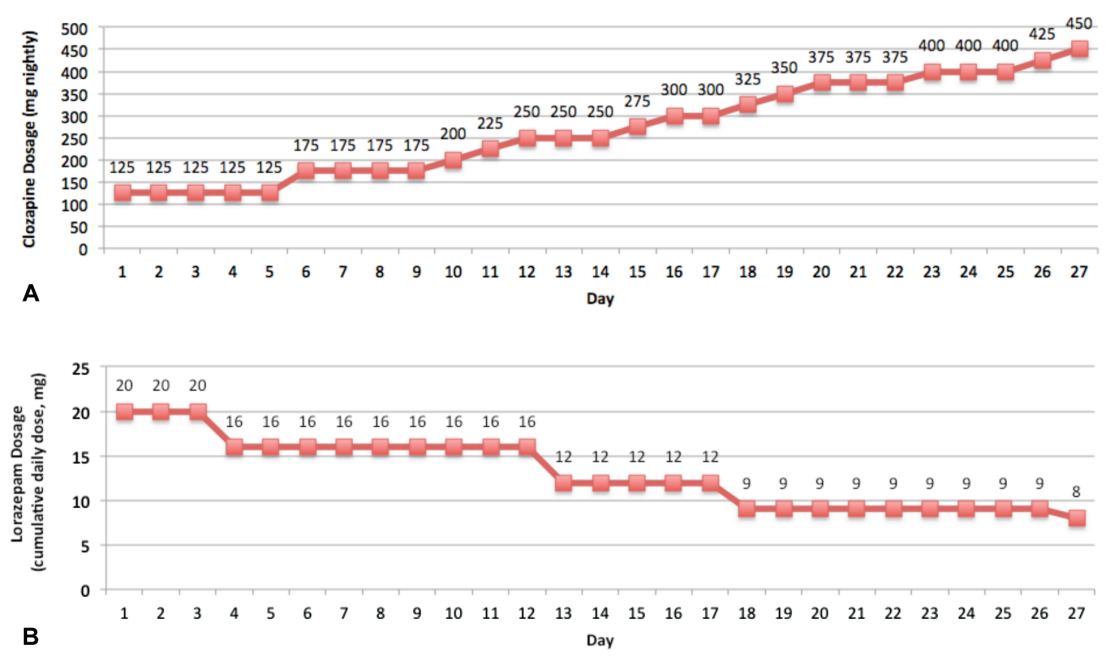

Figure 1. A) Clozapine and B) lorazepam treatment records.

\section{References}

1 Bipeta R, Khan MA. Delirious mania: can we get away with this concept? A case report and review of the literature. Case Rep Psychiatry 2012;2012: 720354.

2. Lee BS, Huang SS, Hsu WY, Chiu NY. Clinical features of delirious mania: a series of five cases and a brief literature review. BMC Psychiatry 2012;12:65.

3. Fink M. Delirious mania. Bipolar Disord 1999;1:54-60.

4. Jung WY, Lee BD. Quetiapine treatment for delirious mania in a military soldier. Prim Care Companion J Clin Psychiatry 2010;12.

5. Oldham MA, Lee HB. Catatonia vis-avis delirium: the significance of recognizing catatonia in altered mental status. Gen Hosp Psychiatry 2015;37:554-9.

6. Wachtel L, Commins E, Park M, et al. Neuroleptic malignant syndrome and delirious mania as malignant catatonia in autism: prompt relief with electroconvulsive therapy. Acta Psychiatr Scand 2015;132:319-20.

7. Luchini F, Medda P, Mariani MG, et al. Electroconvulsive therapy in catatonic patients: efficacy and predictors of response. World J Psychiatry 2015 22;5:182-92.

8. Vasudev K, Grunze H. What works for delirious catatonic mania? BMJ Case Rep 2010;2010.

9. Nicolato R, Costa-Val A, Souza A, et al. Delirious mania associated with bipolar disease in a Brazilian patient: response to ECT and olanzapine. J Neuropsychiatry Clin Neurosci 2009; 21:477.

10. Stroup TS, Gerhard T, Crystal S, et al. Comparative Effectiveness of clozapine and standard antipsychotic treatment in adults with schizophrenia. Am J Psychiatry 2016;173:166-73.

11. Meltzer HY. An overview of the mechanism of action of clozapine. J Clin Psychiatry. 1994;55:47-52. 